\title{
AIRBORNE LIDAR AND HIGH RESOLUTION SATELLITE DATA FOR RAPID 3D FEATURE EXTRACTION
}

\author{
S. D. Jawak ${ }^{\mathrm{a}, *}$, S. N. Panditrao ${ }^{\mathrm{b}}$, A. J. Luis ${ }^{\mathrm{a}}$

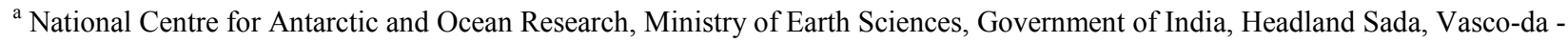 \\ Gama, Goa 403804, India - shridhar.jawak@gmail.com, alvluis1@gmail.com \\ ${ }^{\mathrm{b}}$ Indian National Centre for Ocean Information Services, Ministry of Earth Sciences, Government of India, Ocean Valley, Pragathi \\ Nagar, Nizampet, Hyderabad 500090, India - satej.panditrao@gmail.com
}

KEY WORDS: LiDAR, Feature Extraction, Canopy Height Model (CHM), Accuracy

\begin{abstract}
:
This work uses the canopy height model (CHM) based workflow for individual tree crown delineation and 3D feature extraction approach (Overwatch Geospatial's proprietary algorithm) for building feature delineation from high-density light detection and ranging (LiDAR) point cloud data in an urban environment and evaluates its accuracy by using very high-resolution panchromatic (PAN) (spatial) and 8-band (multispectral) WorldView-2 (WV-2) imagery. LiDAR point cloud data over San Francisco, California, USA, recorded in June 2010, was used to detect tree and building features by classifying point elevation values. The workflow employed includes resampling of LiDAR point cloud to generate a raster surface or digital terrain model (DTM), generation of a hill-shade image and an intensity image, extraction of digital surface model, generation of bare earth digital elevation model (DEM) and extraction of tree and building features. First, the optical WV-2 data and the LiDAR intensity image were co-registered using ground control points (GCPs). The WV-2 rational polynomial coefficients model (RPC) was executed in ERDAS Leica Photogrammetry Suite (LPS) using supplementary *.RPB file. In the second stage, ortho-rectification was carried out using ERDAS LPS by incorporating well-distributed GCPs. The root mean square error (RMSE) for the WV-2 was estimated to be $0.25 \mathrm{~m}$ by using more than 10 well-distributed GCPs. In the second stage, we generated the bare earth DEM from LiDAR point cloud data. In most of the cases, bare earth DEM does not represent true ground elevation. Hence, the model was edited to get the most accurate DEM/ DTM possible and normalized the LiDAR point cloud data based on DTM in order to reduce the effect of undulating terrain. We normalized the vegetation point cloud values by subtracting the ground points (DEM) from the LiDAR point cloud. A normalized digital surface model (nDSM) or CHM was calculated from the LiDAR data by subtracting the DEM from the DSM. The CHM or the normalized DSM represents the absolute height of all aboveground urban features relative to the ground. After normalization, the elevation value of a point indicates the height from the ground to the point. The above-ground points were used for tree feature and building footprint extraction. In individual tree extraction, first and last return point clouds were used along with the bare earth and building footprint models discussed above. In this study, scene dependent extraction criteria were employed to improve the 3D feature extraction process. LiDAR-based refining/ filtering techniques used for bare earth layer extraction were crucial for improving the subsequent 3D features (tree and building) feature extraction. The PAN-sharpened WV-2 image (with $0.5 \mathrm{~m}$ spatial resolution) was used to assess the accuracy of LiDAR-based 3D feature extraction. Our analysis provided an accuracy of $98 \%$ for tree feature extraction and $96 \%$ for building feature extraction from LiDAR data. This study could extract total of 15143 tree features using CHM method, out of which total of 14841 were visually interpreted on PAN-sharpened WV-2 image data. The extracted tree features included both shadowed (total 13830) and non-shadowed (total 1011). We note that CHM method could overestimate total of 302 tree features, which were not observed on the WV-2 image. One of the potential sources for tree feature overestimation was observed in case of those tree features which were adjacent to buildings. In case of building feature extraction, the algorithm could extract total of 6117 building features which were interpreted on WV-2 image, even capturing buildings under the trees (total 605) and buildings under shadow (total 112). Overestimation of tree and building features was observed to be limiting factor in 3D feature extraction process. This is due to the incorrect filtering of point cloud in these areas. One of the potential sources of overestimation was the man-made structures, including skyscrapers and bridges, which were confounded and extracted as buildings. This can be attributed to low point density at building edges and on flat roofs or occlusions due to which LiDAR cannot give as much precise planimetric accuracy as photogrammetric techniques (in segmentation) and lack of optimum use of textural information as well as contextual information (especially at walls which are away from roof) in automatic extraction algorithm. In addition, there were no separate classes for bridges or the features lying inside the water and multiple water height levels were also not considered. Based on these inferences, we conclude that the LiDAR-based 3D feature extraction supplemented by high resolution satellite data is a potential application which can be used for understanding and characterization of urban setup.
\end{abstract}

\section{INTROCUCTION}

Light detection and ranging (LiDAR) is nowadays a very effective and prolific technology as far as detailed mapping and modeling of various types of terrain is concerned. LiDAR is an active remote sensing technology that evaluates properties of reflected light to determine range to a remote object (Lefsky et al., 2002). Airborne LiDAR is capable of providing highly accurate measurements of vertical features with single pulse, multiple pulses, or full waveform. However, its usage is currently limited because of its high acquisition cost. In LiDAR

\footnotetext{
* Corresponding author.
} 
remote sensing, the range to remote object is estimated by computing the time delay between broadcast of a laser pulse and recognition of the reflected signal (Wehr and Lohr, 1999). LiDAR technology is being progressively more practiced in ecology, forestry, geomorphology, seismology, environmental research and remote sensing because of its capability to produce three-dimensional (3D) point data with high spatial resolution and accuracy (Gaveau and Hill, 2003; Brandtberg et al., 2003). LiDAR systems coupled with accurate positioning and orientation systems can obtain precise 3D measurements of earth surface in the form of point cloud data by using high sampling densities. LiDAR is an efficient technique to map and model the vegetation cover and trees located in the landscape. Forest resource management and its impact on various regions is a very critical aspect and LiDAR is a very conducive tool to explore it. Individual tree crown delineation and tree parameter extraction is a complex research topic and various methods have been implemented for this task. LiDAR systems along with precise positioning and orientation systems can obtain highly accurate 3D measurements of earth surface in the form of point cloud data by using high sampling densities (NOAA, 2012). Individual trees extraction is the matter of this study, which is highly important as far as its significant applications in ecology, environmental sciences and forestry are concerned. Various parameters associated with the trees like tree height, crown diameter, canopy based height, diameter at breast height (DBH), biomass, and species type can be determined after individual extraction of trees. There are various traditional methods which include forest inventory, aerial photography interpretation which require intensive field work and large amount of financial support. This can be replaced by airborne LiDAR along with very high resolution satellite imagery, which can also reduce the time consumption, labor and enhance geographical accessibility to a significant extent. LiDAR has been widely applied in forestry (Patenaude et al., 2004; Popescu and Wayne, 2004), and it is found to be useful in mapping individual trees in complex forests (Chen et al., 2007; Kock et al., 2006). Research on exploiting LiDAR point cloud data to evaluate vegetation structures has been progressed from a forest scale to individual tree level. This is evidently encouraged by the developments in LiDAR technology, resulting into higher pulse rates and increased LiDAR posting densities. Therefore, the semiautomatic extraction of single tree (delineation) has become a fundamental approach in forestry research (Heurich, 2008). Computing tree attributes at high spatial scales is essential to monitor terrestrial natural resources (Zimble et al., 2003). However, not many studies have focused on individual tree level feature extraction. One of the main challenges of this research is result validation and accuracy assessment for individual extracted tree measurements, where detailed field inventory and/or very high resolution satellite image is necessary. The high spatial density LiDAR point cloud data noticeably revealed the structure of individual trees, and hence provided better prospect for more accurate tree feature extraction and vegetation structure parameters. The high density LiDAR has been successfully employed to demarcate the whole structure of individual tree (Rahman, and Gorte, 2009; Reitberger, 2007). There are numerous methods proposed to demarcate individual trees using airborne LiDAR point cloud data. Popescu and Wynne (2004) employed a local maximum filtering method to extract individual trees. Tiede et al. (2005) practiced a similar local maximum filtering method to recognize tree tops and developed a region growing algorithm to extract tree features. Chen et al. (2007) proposed a watershed segmentation to isolate individual trees, where the tree tops extracted by local maxima were used as markers to improve the accuracy. Koch et al. (2006) extracted tree features by synergetic usage of pouring algorithm and knowledge based assumptions on the structure of trees. Korpela et al. (2007) used a multi-scale template matching approach for tree feature extraction using elliptical templates to represent tree models. Falkowski et al. (2006) proposed the spatial wavelet analysis to semiautomatically verify the spatial location, height, and crown diameter of individual tree features from LiDAR point cloud data. These algorithms extract individual tree features using the LiDAR derived canopy height model (CHM). CHM is a raster image interpolated from LiDAR cloud points indicating the top of the vegetation canopy. Tree detection and tree crown delineation from Airborne LiDAR has been focusing mostly on utilizing the CHM. However, CHM can have inherent errors and uncertainties, e.g. spatial error introduced during the interpolation from the point cloud to raster (Guo et al., 2007), which may reduce the accuracy of tree feature extraction. Therefore, new methods to delineate individual tree features from the LiDAR point cloud necessitate development and validation. Morsdorf et al. (2004) employed the $k$-mean clustering algorithm to delineate individual tree features from the point cloud, but their accuracy depended on seed points extracted from the local maxima of a digital surface model. 3D building models are essential for many Geographic Information System (GIS) applications such as urban planning, disaster management and city planning (Awrangjeb et al., 2013; Gröger and Plümer, 2012). Therefore, 3D building extraction has been an area of vigorous research within the photogrammetric, remote sensing and computer vision communities for the last two decades. Detailed and up-to-date building information is of enormous importance to every resident, government agencies, and private companies (e.g. real estates). Remote sensing (RS) is one of the most professional ways to obtain and extract the required geographical information (Jensen and Cowen, 1999). However, the traditional manual digitization for building extraction using raw imagery is highly labor-intensive, timeconsuming and expensive. During the past two decades many researchers in photogrammetry, remote sensing and computer vision communities have been trying to study and develop the automatic or semi-automatic approaches for building extraction and reconstruction (Mayer, 1999). For monocular image, shadow analysis is often used to assist building detection. In this study, we used method for individual tree delineation based on canopy height model (CHM) and 3D feature extraction approach (Overwatch Geospatial's proprietary algorithm) for building feature delineation from the high resolution airborne LiDAR point cloud data. To investigate the effectiveness of these methods in extracting individual trees and buildings, we used very high resolution remote sensing data from WorldView-2 (WV-2) satellite. This study aims to assess the accuracy of individual tree and building extraction using LiDAR by visual interpretation of trees onto very high resolution WV-2 image.

\section{STUDY AREA AND DATA}

In order to investigate and illustrate the effectiveness of LiDAR based tree and building feature extraction, we selected the part of San Francisco city, California, United States of America (37 $44^{\prime \prime} 30 \mathrm{~N}^{\prime}, 122^{\circ} 31^{\prime \prime} 30^{\prime} \mathrm{W}$ and $37^{\circ} 41^{\prime \prime} 30^{\prime} \mathrm{N}, 122^{\circ} 20^{\prime \prime} 30^{\prime} \mathrm{W}$ ), as a test scene. San Francisco is situated on the West Coast of the USA at the north ending of the San Francisco Peninsula and comprises of significant extension of the Pacific Ocean and San Francisco Bay within its margins. The mainland area within the 
city constitutes roughly $600 \mathrm{~km}^{2}$. There are more than 50 hills within city boundaries. There are more than 220 parks maintained in the San Francisco by Recreation \& Parks Department, containing thousands of native trees and plants.

We used the standard airborne LiDAR data over San Francisco, California, USA, recorded in June 2010. The data was in LASer (LAS) (Figure 1). In addition to airborne LiDAR data, we also used radiometrically-corrected, geo-referenced, orthorectified 16-bit standard level 2 (LV2A) WV-2 multi-sequence datasets, including single band PAN and 8-band MSI images at $46 \mathrm{~cm}$ and $185 \mathrm{~cm}$ ground sample distance, which were resampled to $50 \mathrm{~cm}$ and $200 \mathrm{~cm}$, respectively. Level $2 \mathrm{~A}$ of image preprocessing had been done by the DigitalGlobe. The images were acquired during nearly cloud-free bright illumination on $9^{\text {th }}$ October 2011 over San Francisco covered a number of buildings, vegetation structures, forest structures, skyscrapers, industrial structures, residential houses, highways, community parks, and private housing (Figure 2). The calibration metadata was used to convert the raw digital numbers to radiance. Necessary pre-processing methods like Data Calibration, Darkpixel Subtraction, PAN-sharpening etc. were employed for better visualization.

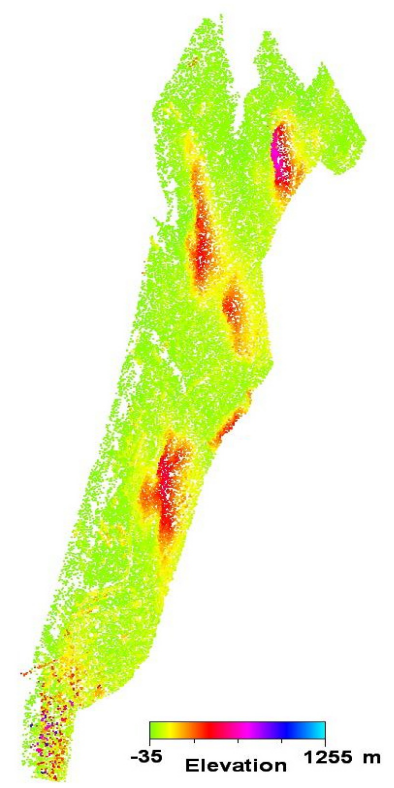

Figure 1. LiDAR based point cloud representation over the extent of study area.

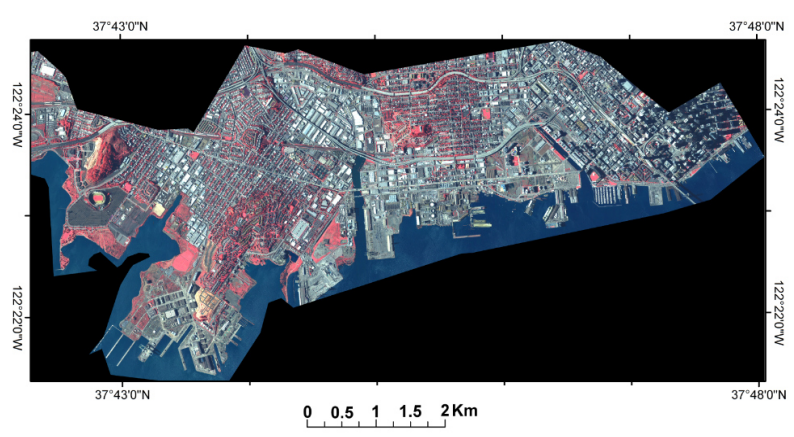

Figure 2. WorldView-2 PAN-sharpened satellite image over the study area.
The remote sensing (RS) data cannot be used efficiently without ground truth, especially for urban studies. The successful interpretation of RS data requires supplementary field work to understand the small-scale variations that are common in urban land cover. PAN-sharpened WV-2 $(0.5 \mathrm{~m})$ supplemented by publicly available GIS maps and historical Google Earth images were used for accuracy analysis.

\section{METHODOLOGY}

Preprocessing of the WV-2 imagery comprises of four separate steps: (i) data preparation, (ii) Data fusion, (iii) Co-registration of WV-2 Pan-sharpened images to the LiDAR data, and (iv) Shadow compensation using LiDAR based DSM (Jawak and Luis, 2014a; Jawak and Luis, 2014b). First, a dark pixel subtraction was performed to reduce the path radiance from each band. The dark object is the minimum digital number (DN) value for more than 1000 pixels over the whole image (Jawak and Luis, 2013a; Jawak and Luis, 2013b; Jawak and Luis, 2013c; Jawak and Luis, 2013d). The calibration procedure was carried out in two steps: (i) conversion of the raw DN values to at-sensor spectral radiance factors and (ii) conversion from spectral radiance to Top-of-Atmosphere (TOA) reflectance (Jawak and Luis, 2012; Jawak and Luis, 2011). In order to create an image at $0.50 \mathrm{~m}$ resolution, the multiband image was PAN-sharpened from a resolution of $2.00 \mathrm{~m}$ to 0.50 $\mathrm{m}$ by using Hyperspherical Color Sharpening (HCS) fusion method which has been specifically developed for the WV-2 data (Jawak et al., 2013a). The first and possibly the most important precursor step of the tree and building feature extraction process is the precise co-registration between all datasets. In fact, neglecting geo-registration can lead to false accuracy analysis. The optical WV-2 data and the LiDAR intensity image were co-registered. Co-registration was performed in two steps: (i) geometric correction without ground control points (GCPs) and (ii) ortho-rectification using ground control points. The main challenge was to match the resolution of LiDAR intensity image with the resolution of PANsharpened WV-2 image. At first, the WV-2 rational polynomial coefficients model (RPC) was executed in ERDAS Leica Photogrammetry Suite (LPS) using supplementary .RPB file. In the second stage, ortho-rectification was carried out using ERDAS LPS by incorporating well-distributed GCPs. The obtained root mean square error (RMSE) for the WV-2 was estimated to be $0.25 \mathrm{~m}$ using more than 10 well-distributed GCPs.

LiDAR-based individual tree and building feature extraction consists of five main tasks: (1) bare earth digital elevation/terrain (DEM/DTM), DSM, and intensity image generation, (2) building footprint extraction, (3) individual trees/vegetation/forest extraction using $\mathrm{CHM}$ and building extraction using 3D fetaure extraction approach, (4) tree filtering, and (5) accuracy assessment of tree and building feature extraction. Many different software packages are available to resample LiDAR point clouds into 2-D grids and advanced processing. We utilized Overwatch system's LIDAR Analyst for ArcGIS, LAStools $(\mathcal{C}$ software, and Qcoherent software LP360 for ArcGIS. Conversion of point clouds to uniform raster surfaces or 2D-grids by resampling methods is the first essential step in many LiDAR based applications. Many surface interpolation methods are available in literature for effective rasterization (Gurram et al., 2013). The choice of cell size affects the quality of 2D- raster models or surfaces generated. We selected a grid size of $50 \mathrm{~cm}$ to match the $50 \mathrm{~cm}$ 
resolution of PAN-sharpened WV-2 image and based on average tree diameters interpreted using WV-image. A bare earth DEM/DTM, a DSM and an intensity image were derived from the raw airborne LiDAR data. The DEM/DTM was generated by triangulating elevation values only from the bareearth LiDAR points, while the DSM was generated by triangulating elevation only from the first-return LiDAR points (Figure 3). The intensity image was generated by triangulating intensity from the first-return LiDAR points. Surfacing was used to interpolate the ground points and generate the DEM (Guo et al., 2010). In this study, the ground points were collected and interpolated using an adaptive triangulated irregular network (TIN) model. We employed TIN interpolation method over IDW and spline, because the LiDAR point cloud data was very dense and spline and IDW method failed to give desired results. TIN approach also considers the density variation between data points. As the study area is urban, this method provided good results when compared to other methods like Kriging which is useful in the areas consisting of diverse features which exhibit high degree of spatial auto-correlation. Bare earth DTM/DEM extraction is followed by editing or cleaning of that bare earth layer. In most of the cases, bare earth DEM does not represent true ground elevation. Hence, the model was cleaned/ edited to get the most accurate DEM/ DTM possible. After DTM editing, we normalized the LiDAR point cloud data based on DTM in order to reduce the effect of undulating terrain. The normalization step is very significant since the tree filtering algorithm needs to define a reference height for further processing. We normalized the vegetation point cloud values by subtracting the ground points (DEM) from the LiDAR point cloud. A normalized digital surface model (nDSM) or CHM is calculated from the LiDAR data by subtracting the DEM from the DSM. The CHM or the normalized DSM represents the absolute height of all aboveground urban features relative to the ground. After normalization, the elevation value of a point indicates the height from the ground to the point. The above-ground points were used for tree feature and building footprint extraction. The second step of the workflow is to identify building measurements from non-building (mainly vegetation). Building footprint extraction consists of extracting the footprints of buildings in 3D shapefile format along with the attribute table showing the information about each building polygon. This task also consists of editing building footprint layer so as to separate the merged buildings. We employed LiDAR analyst 4.2 for ArcGIS workflow for buiding extraction. The parameters used for building extraction are listed in Table 1, 2 and 3. The final output map of building feature extraction is shown in Figure 4. We used the CHM (Jawak et al., 2013b) based method for individual tree feature extraction. The individual tree extraction based on this method produces 3D shapefile for extracted tree features. It is generally assumed that the LiDAR points other than the terrain are tree features in the urban areas. The calculation of individual tree height is difficult because it is indistinct where the laser pulse hits and be reflecting on the tree. A local maximum filtering with variable search window approach was used to detect tree features. In individual tree extraction, first and last return point clouds were used along with the bare earth and building footprint models discussed above. While extracting the trees, the minimum tree height was set to a value $(0.5 \mathrm{~m})$ that corresponds with the size of vegetation we desire to be called a tree. The resulting shapefile of tree feature extraction consists of point features showing individual trees. While extracting forests, maximum distance between the trees and minimum size of group of the trees/ minimum size of a forest were specified by trial and error method to achieve desired results. After tree feature extraction, LiDAR point cloud classification was performed so as to classify different points according to their elevation values and defined criteria. The accuracy of the classification highly depends on the user defined criteria (Table 1 and 2). Texture variance for trees and minimum difference between returns for trees are the crucial parameters which affect the extraction accuracy. The classified LiDAR output contained three categories: bare earth, buildings and vegetation. The text file of output gives information about the total points included or excluded in a particular class, maximum height, minimum height, etc (Table 3). The segmented point clouds and the tree locations are then used as input for tree filtering routine. Since the data includes many elevated objects such as buildings, trees and bridges, the classification or filtering is needed in various LiDAR applications. The classification of point cloud data is called the filtering process of LiDAR data. In this study, a tree filtering algorithm aims at separating dominant trees and aboveground objects such as buildings, bridges and undergrowth vegetation. This algorithm requires three input parameters: 1) maximum growing distance for tree crown, 2) maximum growing distance for tree trunk, and 3) average tree trunk diameter. The final output map of tree feature extraction is shown in Figure 5. In accuracy assessment, we visually interpreted the LiDAR-classified trees on WV-2 image. The 8band WV-2 image was georeferenced, orthorectified and PANsharpened using HCS method. WV-2 PAN-sharpened image $(0.5 \mathrm{~m})$ was used such that the tree and building features can be easily recognized. The PAN-sharpened image $(0.5 \mathrm{~m})$ was visualized in ArcGIS 10 at several scales for the better visualization of tree features using various band combinations: 7-4-2; 8-7-2; 6-3-2; 5-3-2 and 7-3-2. Finally, 1:500 scale and 53-2 band combination was selected for visualization of the tree and building features. Based on publically available map datasets and Google images of the study area, the WV-2 HCSsharpened image was manually evaluated using ArcGIS 10 to visualize against LiDAR based extracted tree and building features. All the tree and building features extracted by processing LiDAR data were evaluated using WV-2 PANsharpened image to interpret individual trees and buildings for statistical accuracy assessment.

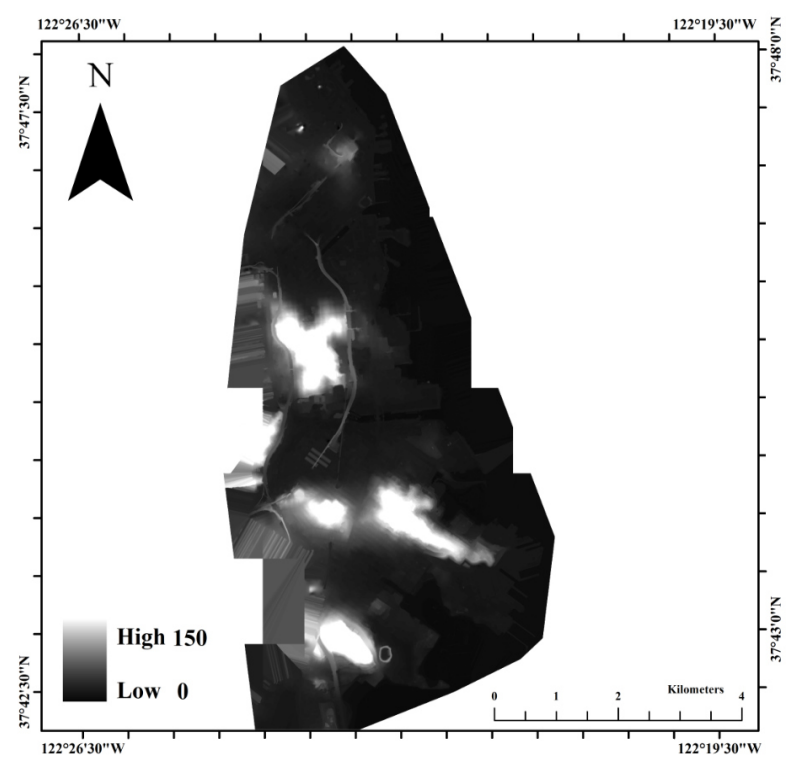

Figure 3. LiDAR Bare Earth DEM (scale in meter) 


\begin{tabular}{ll}
\hline Processing Steps & Criteria \\
\hline Bare Earth extraction method & Point Clouds \\
Return filter & All returns \\
Remove buildings with area more than & $35000 \mathrm{~m}^{2}$ \\
Minimum slope for building roofs & $15^{\circ}$ \\
Maximum slope for building roofs & $40^{\circ}$ \\
Texture variance for trees & $80 \%$ \\
Smoothing tolerance & $1 \mathrm{~m}$ \\
Minimum height for tall buildings & $15 \mathrm{~m}$ \\
Minimum area for tall buildings & $200 \mathrm{~m}^{2}$ \\
Tree extraction method & Variable \\
& window search \\
Predominant tree/forest type & Mixed \\
Minimum tree height & $3 \mathrm{~m}$ \\
Maximum tree height & $40 \mathrm{~m}$ \\
Minimum size of the forest & $600 \mathrm{~m}^{2}$ \\
\hline
\end{tabular}

Table 1. Criteria used for bare earth, buildings and trees extraction

\begin{tabular}{lc}
\hline \multicolumn{1}{c}{ Processing Steps } & Criteria \\
\hline Ground height threshold & $0.3 \mathrm{~m}$ \\
Minimum height (Building settings) & $1.5 \mathrm{~m}$ \\
Search Radius & $2 \mathrm{~m}$ \\
Minimum height for Low vegetation & $0.5 \mathrm{~m}$ \\
Minimum height for medium Vegetation & $1 \mathrm{~m}$ \\
Minimum height for high vegetation & $2 \mathrm{~m}$ \\
\hline
\end{tabular}

Table 2. Criteria for point cloud classification.

\begin{tabular}{|c|c|c|c|c|}
\hline \multirow[t]{2}{*}{ Parameter } & \multicolumn{2}{|c|}{ Building Features } & \multicolumn{2}{|c|}{ Tree Features } \\
\hline & Included & Excluded & Included & Excluded \\
\hline Min. Height & 1.50 & 0.50 & 1.00 & 0.50 \\
\hline $\begin{array}{l}\text { Max. } \\
\text { Height }\end{array}$ & 406.54 & 1.50 & 34.19 & 1.00 \\
\hline $\begin{array}{l}\text { Mean } \\
\text { Height }\end{array}$ & 13.00 & 0.92 & 5.92 & 0.72 \\
\hline Point Count & 13999456 & 331429 & 7315433 & 1718478 \\
\hline
\end{tabular}

Table 3. LAS classification statistics for building and tree class (height statistics for LAS points that were classified as a given feature class are recorded in the "Included" field, and statistics in the "Excluded" field were gathered from points that should have been classified as the given feature class but were weeded out due to the user-defined classification settings)

\section{RESULTS}

Our research focus on CHM-based tree feature extraction and LiDAR analyst's 3D building fetaure extarction using highresolution airborne LiDAR data and its accuracy assessment using high-resolution WV-2 image data. In building and tree feature extraction methodology, the scene dependent criteria were used. Texture variance for tree features and minimum difference between returns for trees were the crucial parameters in building and trees feature extraction. The results depicted in Table 3 show that all the LiDAR points exihibit point cloud classification and hence, the criteria used for tree and building feature extraction were appropriate. A 8-band WV-2 image was used for visual interpretation of LiDAR-classified tree and building features. The image was PAN-sharpened and georeferenced which helped in proper visualization of LiDAR tree points. The results depicted in Table 4 shows that 15143 tree features were extracted by CHM method using LiDAR point cloud data. All the tree features extracted using LiDAR data were cross-verified using multitemporal high resolution images, which indicates that the 14841 tree features were correctely interepreted out of 15143 tree features. The overall accuracy of LiDAR based tree feature extraction was found to be $98 \%$ against the high resolution satellite image as a reference. A DSM-based shadow mask was used for reducing the potential source of error attributable to topography-based shadow in high resolution image. A total of 1011 tree features under shadow were cross-verified using multitemporal image data.

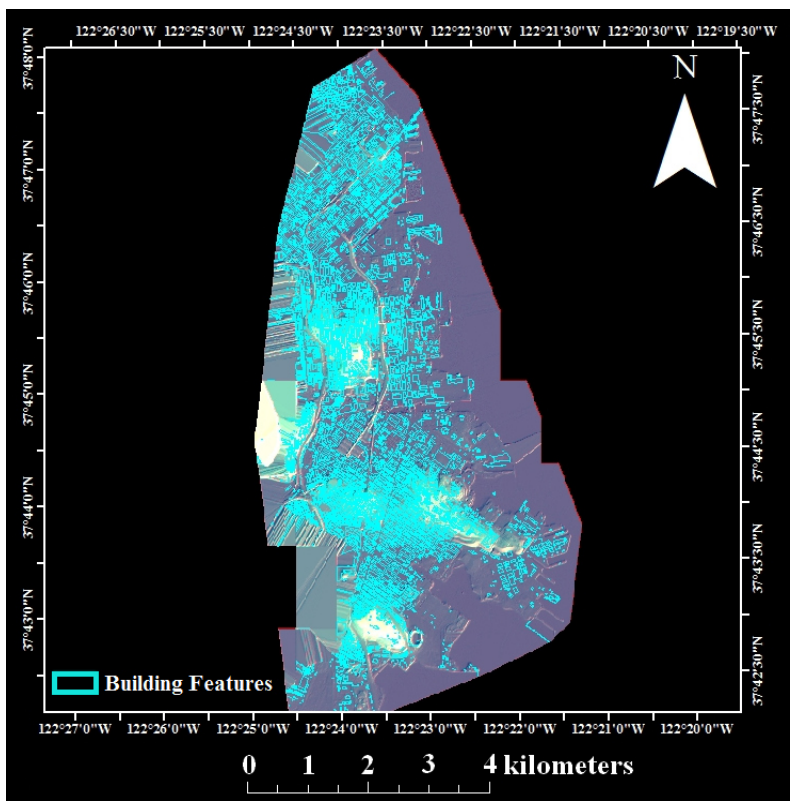

Figure 4. The final output map of building feature extraction

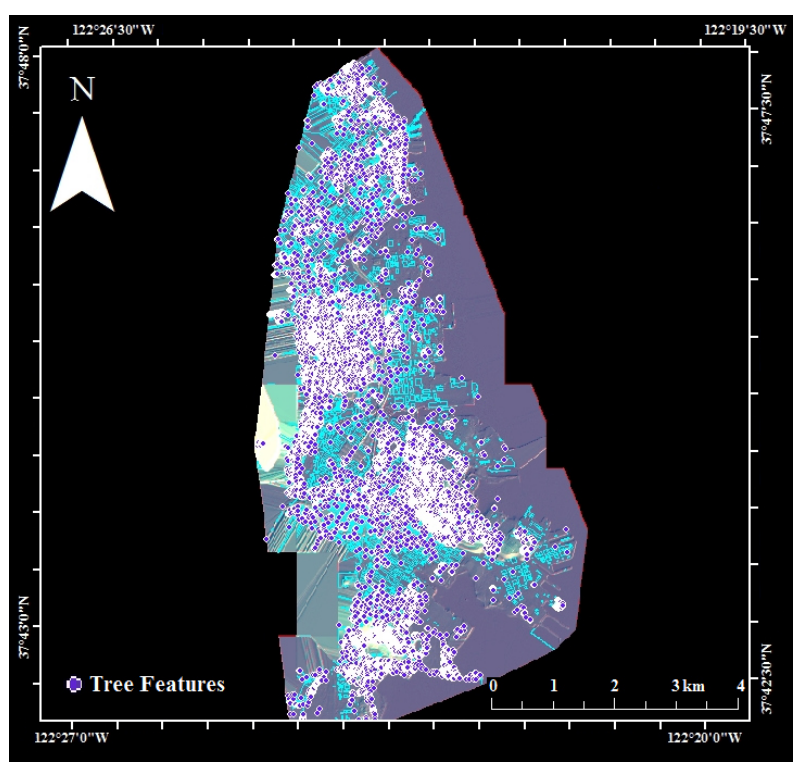

Figure 5. LiDAR-based tree feature extraction

It is evident that the LiDAR-based extraction caused overestimation of 302 tree features, which can be attributed to two methodological and experimental inadequacies: 1) the present research was carried out using scene dependent criteria which should be optimized with trail-and-error method, and 2) the 
error might be propogated during the LiDAR filtering or classification process. For optimal tree feature extraction, we propose the rigorous optimization of criteria and synergetic usage of high resolution data for tree feature extraction in future studies. LIDAR Analyst's 3D extraction approach is used for collecting features and generating raster data from airborne LiDAR. It simplifies the process of extracting bare earth, buildings, and trees/forests features.

\begin{tabular}{ll}
\hline ACCURACY ATTRIBUTE & VALUE \\
\hline $\begin{array}{l}\text { Total tree features extracted from LiDAR by } \\
\text { using CHM method }\end{array}$ & 15143 \\
$\begin{array}{l}\text { Total tree features visually interpreted using } \\
\text { multitemporal image data }\end{array}$ & 14841 \\
$\begin{array}{l}\text { Total non-shadowed tree features visually } \\
\text { interpreted using multitemporal image data }\end{array}$ & \\
Total shadowed tree features visually & 1011 \\
interpreted using multitemporal image data & \\
$\begin{array}{l}\text { Total overestimated tree features } \\
\text { Overall accuracy for tree feature extraction }\end{array}$ & 302 \\
\hline
\end{tabular}

Table 4. Statistics for LiDAR based tree feature extraction accuracy results

\begin{tabular}{ll}
\hline ACCURACY ATTRIBUTE & VALUE \\
\hline $\begin{array}{l}\text { Total building features extracted from LiDAR by } \\
\text { using 3d feature extraction method }\end{array}$ & 6370 \\
$\begin{array}{l}\text { Total building features visually interpreted using } \\
\text { multitemporal image data }\end{array}$ & 6117 \\
$\begin{array}{l}\text { Total non-shadowed building features visually } \\
\text { interpreted using multitemporal image data }\end{array}$ & 6005 \\
$\begin{array}{l}\text { Total shadowed building features visually } \\
\text { interpreted using multitemporal image data }\end{array}$ & 112 \\
$\begin{array}{l}\text { Total building features which are hidden under } \\
\text { trees }\end{array}$ & 605 \\
$\begin{array}{l}\text { Total building features which are not hidden } \\
\text { under trees }\end{array}$ & 5512 \\
$\begin{array}{l}\text { Total overestimated building features } \\
\text { Overall accuracy for building feature extraction }\end{array}$ & $96 \%$ \\
\hline
\end{tabular}

Table 5. Statistics for LiDAR based building feature extraction accuracy results

The results depicted in Table 5 show that 6370 building features were extracted by 3D fetaure extraction method using LiDAR point cloud data. All the building features extracted using LiDAR data were cross-verified using multitemporal high resolution images, which indicates that the 6117 building features were correctly interpreted out of 6370 building features. The overall accuracy of LiDAR based building feature extraction was found to be $96 \%$ against the high resolution satellite image as a reference. In addition to visual interpretation of all the extracted buildings, we randomly sampled 250 points within areas classified as buildings and another 250 points from areas outside those regions classified as buildings to visually determine the number of points within the building areas that were correctly and incorrectly classified. The same procedure was done for region outside the building areas. The building extraction process yielded 96\% Overall accuracy with 98\% Producer's accuracy and 95\% User's accuracy.

\section{DISCUSSION}

Separation of point cloud into ground and non-ground is the most critical step for DEM / DTM generation from point cloud data. Filtering and interpolation algorithms play a major role in this task. As point cloud is able to penetrate the forested areas, it has an advantage over photogrammetry of a highly accurate DTM extraction in forested areas. Numerous methods have been developed for point cloud processing so far, but some more work has to be done to get much better results. High density datasets make it easy to filter ground from non-ground points, but for low density datasets, choice of filtering algorithm is an utmost important step to achieve the highest possible accuracy. Due to significant increase in the volume in case of highly dense point cloud data, data storage, processing and manipulation have become important issues to be taken care of. We note that the use of a PAN-sharpened image as reference data for the accuracy analysis introduces, to some extent, data circularity. We cross-verified the tree and building features visually interpreted on PAN-sharpened WV-2 images with multitemporal satellite image data to reduce data circularity and bias due to visual interpretation of WV-2 data. Additionally, we have carried out an extensive accuracy analysis of the tree and building feature extraction using the visual interpretation of WV-2 image supported by empirical cross-verification of tree and building points in terms of visual analysis of Google Earth images of the study area and employing different methods using various sources of ground reference data acquired through several means from urban areas, which are publicly available (GIS-based) as maps and manually prepared polygons. We also note that the difference in acquisition of WV-2 and LiDAR datasets might have affected the analysis, however, this study employs many supplementary temporal datasets in the analysis. Therefore, we surmise that the potential data circularity existing in our accuracy analysis had a relatively insignificant effect on the comparative performance of the tree feature extraction. It is noted that tree features are over estimated which are adjacent to building features, and it could have reduced if we had subtracted building layer and then carry out the accuracy analysis. However, we are testing the inherent capability of LiDAR data to extract tree features using CHM. Subtracting building feature layer would produce the biased accuracy analysis. Hence, we carried out the accuracy analysis without subtracting the building layer. We also note that the present attempt should be compared with other existing methods for extracting tree and building features. However, the present study is much focused on existing algorithms for extracting tree and building features and cross-verifying the outcomes using high resolution satellite images. The present study insinuates some future studies for comparison of $3 \mathrm{D}$ feature extraction methods using high resolution satellite data as a reference.

\section{CONCLUSION}

The high resolution airborne LiDAR data provides tremendous potential for tree and building feature extraction in urban landscape and the hyperspatial WV-2 imagery supplements the accuracy assessment procedure. The objective of this study was to evaluate CHM-based tree-feature and building feature extraction by LiDAR analyst's accuracy by visual interpretation/ identification on 8-band WV-2 image. Our study uses the algorithm developed by Overwatch system's LiDAR Analyst for ArcGIS for LiDAR feature extraction and classification with scene dependent criteria. The 8-band WV-2 image provides better recognition and extraction of various land-cover features 
and due to the inclusion of new bands in the imagery, the vegetation analysis becomes more effective. The present study provides following conclusions. (1) Texture variance for trees and minimum difference between returns for trees turned out to be the two most important factors in discriminating the tree and building features in the LiDAR data. (2) Preprocessing of the WV-2 image improved the visualization of vegetation features. (3) LiDAR data was found to be capable of extracting shadowcovered tree and building features. (4) LiDAR point cloud data can be used in conjunction with satellite image data for supporting tree and building feature extraction. The research highlights the usefulness of the commonly used methodology for the LiDAR data processing and the effectiveness of 8-band WV-2 remotely sensed imagery for accuracy assessment of LiDAR-based tree and building feature extraction capability.

\section{ACKNOWLEDGEMENT}

We are indebted to the organizers of the IEEE GRSS Data Fusion Contest (2012), who provided the data to our research team free of cost. We also acknowledge Dr. Rajan, Director, NCAOR, Dr. R. Ravindra, ex-Director, NCAOR, and Prof. Anjana Vyas, CEPT University for their encouragement and motivation for this research. This is NCAOR contribution no. $40 / 2014$.

\section{REFERENCES}

Awrangjeb, M., Zhang, C., Fraser, C. S., 2013. Automatic extraction of building roofs using LIDAR data and multispectral imagery. ISPRS Journal of Photogrammetry and Remote Sensing, 83, pp. 1-18.

Brandtberg, T., Warner, T.A., Landenberger, R.E., J.B. McGraw., 2003. Detection and analysis of individual leaf-off tree crowns in small footprint, high sampling density lidar data from the eastern deciduous forest in North America. Remote Sensing of Environment, 85(3), pp 290-303.

Chen, Q., Gong, P., Baldocchi, D., Tian, Y.Q., 2007. Estimating basal area and stem volume for individual trees from lidar data. Photogrammetric Engineering \& Remote Sensing, 73(12), pp. 1355-1365

Falkowski, M.J., Smith, A.M.S., Hudak, A.T., Gessler, P.E., Vierling, L.A., Crookston, N.L., 2006. Automated estimation of individual conifer tree height and crown diameter via twodimensional spatial wavelet analysis of lidar data. Canadian Journal of Remote Sensing, 32, pp.153-161.

Gaveau, D.L.A., Hill, R.A., 2003. Quantifying canopy height underestimation by laser pulse penetration in small-footprint airborne laser scanning data. Canadian Journal of Remote Sensing, Vol. 29, No. 5, pp 650-657.

Gröger, G., Plümer, L., 2012. Citygml - interoperable semantic $3 \mathrm{~d}$ city models. ISPRS Journal of Photogrammetry and Remote Sensing 71 (7), 12-33.

Guo, Q., Kelly, M., Gong, P., Liu, D., 2007. An object-based classification approach in mapping tree mortality using high spatial resolution imagery. GIScience \& Remote Sensing, 44(1) pp. 24-47.
Guo, Q., Li, W., Yu, H., Alvarez, O., 2010. Effects of Topographic Variability and Lidar Sampling Density on Several DEM Interpolation Methods. Photogrammetric Engineering \& Remote Sensing, 76(6), pp. 701-712.

Gurram, P., Hu, S., Chan. A., 2013. Uniform grid upsampling of 3D lidar point cloud data. SPIE 8650, Three-Dimensional Image Processing (3DIP) and Applications (ed by, p. 86200B)

Heurich, M., 2008. Automatic recognition and measurement of single trees based on data from airborne laser scanning over the richly structured natural forests of the Bavarian Forest National Park. Forest Ecology and Management, 255(7), pp. 2416-2433.

Jawak, S.D., Luis, A.J., 2013a. Improved land cover mapping using high resolution multi-angle 8-band WorldView-2 satellite remote sensing data. Journal of Applied Remote Sensing, 7(1), 073573 .

Jawak, S.D., Luis, A.J., 2013b. A spectral index ratio-based Antarctic land-cover mapping using hyperspatial 8-band WorldView-2 imagery. Polar Science, 7(1), pp. 18-38.

Jawak, S.D., Luis, A.J., 2013c. Very-high resolution remotely sensed satellite data for improved land cover extraction of Larsemann Hills, east Antarctica. Journal of Applied Remote Sensing, 7(1), 073460. doi: 10.1117/1.JRS.7.073460

Jawak, S.D., Luis, A.J., 2013d. A comprehensive evaluation of PAN-sharpening algorithms coupled with resampling methods for image synthesis of very high resolution remotely sensed satellite data. Advances in Remote Sensing, 2(4), pp. 332-344.

Jawak, S.D., Luis, A.J., 2014a. A semiautomatic extraction of Antarctic lake features using WorldView-2 imagery. Photogrammetric Engineering \& Remote Sensing 80(10), pp. $33-46$.

Jawak, S.D., Luis, A.J., 2014b. A Novel Set of Normalized Difference Snow/Ice Index ratios for Extraction of Snow Cover from the Antarctic Environment using Very High Resolution Satellite Data, XXXIII SCAR and $6^{\text {th }}$ Open Science Conference, Auckland, New Zealand, pp. 912.

Jawak, S.D., Luis, A.J., Panditrao, S.N., Khopkar, P.S., Jadhav, P.S., 2013a. Advancement in landcover classification using very high resolution remotely sensed 8-band WorldView-2 satellite data. International Journal of Earth Sciences and Engineering. 6(2), pp. 1742-1749

Jawak, S. D., Panditrao, S. N., A. Luis, 2013b. Validation of High-Density Airborne LiDAR-Based Feature Extraction Using Very High Resolution Optical Remote Sensing Data. Advances in Remote Sensing, 2(4) pp. 297-311.

Jawak, S.D., Luis, A.J., 2012. WorldView-2 satellite remote sensing data for polar geospatial information mining of Larsemann Hills, East Antarctica. In: Proceedings of $11^{\text {th }}$ Pacific (Pan) Ocean Remote Sensing Conference (PORSEC), Id: PORSEC2012-24-00006, Kochi, Kerala, India,

Jawak, S.D., Luis, A.J., 2011. Applications of WorldView-2 satellite data for extraction of polar spatial information and DEM of Larsemann Hills, East Antarctica. International 
Conference on Fuzzy Systems and Neural Computing (FSNC 2011), 3, pp. 148-151.

Jensen, J. R., D. C. Cowen., 1999. Remote sensing of urban/suburban infrastructure and socio-economic attributes. Photogrammetric Engineering \& Remote Sensing, 65(5), pp. 611-622.

Koch, B., Heyder, U., Weinacker, H., 2009. Detection of individual tree crowns in airborne lidar data. Photogrammetric Engineering \& Remote Sensing, Vol. 72, No. 4, pp. 357-363.

Korpela, I., Dahlin, B., Schäfer, H., Bruun, E., Haapaniemi, F., Honkasalo, J., Ilvesniemi, S., Kuutti, V., Linkosalmi, M., Mustonen, J., Salo, M., Suomi, O., Virtanen, H., 2007. Singletree forest inventory using lidar and aerial images for 3D treetop positioning, species recognition, height and crown width estimation. International Archives of Photogrammetry, Remote Sensing and Spatial Information Sciences (IAPRS), XXXVI, pp. 227-233.

Lefsky, M.A., Cohen, W.B., Parker G.G., D.J. Harding., 2002. Lidar remote sensing for ecosystem studies. BioScience, 52(1), pp. 19-3.

Mayer, H., 1999. Automatic object extraction from aerial imagery: a survey focusing on buildings. Computer Vision and Image Understanding, 74(2), pp. 138-149.

Morsdorf, F., Meier, E., Kötz, B., Itten, K. I., Dobbertin, M., Allgowe, B., 2004. LIDAR-based geometric reconstruction of boreal type forest stands at single tree level for forest and wildland fire management. Remote Sensing of Environment, Vol. 92, No. 3, pp. 353-362.

National Oceanic and Atmospheric Administration (NOAA) Coastal Services Centre. 2012. Lidar 101: An Introduction to Lidar Technology, Data, and Applications. Revised. Charleston, SC: NOAA Coastal Services Centre.

Patenaude, G., Hill, R.A., Milne, R., Gaveau, D.L.A., Briggs, B.B.J, Dawson, T.P., 2004. Quantifying forest above ground carbon content using LiDAR remote sensing. Remote Sensing of Environment, Vol. 93, No. 3, pp. 368-380.

Popescu, S.C., Wynne, R.H., 2004. Seeing the trees in the forest: using lidar and multispectral data fusion with local filtering and variable window size for estimating tree height. Photogrammetric Engineering \& Remote Sensing, 70(5), pp. 589-604.

Rahman, M.Z.A., and Gorte, B.G.H., 2009. A new method for individual tree delineation and undergrowth removal from high resolution airborne LiDAR. Proceedings ISPRS Workshop Laserscanning 2009, Paris, France, IAPRS, XXXVIII (3/W8), Vol. XXXVIII, pp.123-128.

Reitberger, J., Heurich, M., Krzystek, P., Stilla, U., 2007. Single tree detection in forest areas with high-density LiDAR data. International Archives of Photogrammetry, Remote Sensing and Spatial Information Sciences, Vol. 36, pp. 139144.

Tiede, D., Hochleitner, G., Blaschke, T., 2005. A full GISbased workflow for tree identification and tree crown delineation using laser scanning. Proceedings of CMRT 05, International Archives of Photogrammetry and Remote Sensing (U. Stilla, F. Rottensteiner, and S. Hinz, editors), XXXVI(Part 3/W24), Vienna, Austria.

Wehr, A., Lohr, U., 1999. Airborne laser scanning-an introduction and overview. ISPRS Journal of Photogrammetry and Remote Sensing, Vol. 54, No. 2-3, pp 68-82.

Zimble, D.A., Evans, D.1., Calrson, G.C., Parker, R.C., 2003. Characterizing vertical forest structure using small-footprint airborne LiDAR. Remote Sensing of Environment, Vol. 87, pp. 171-182. 